\title{
New constraints on the surface of Pluto ${ }^{\star}$
}

\author{
F. Merlin

\begin{abstract}
LESIA-Observatoire de Paris, CNRS, UPMC Univ Paris 06, Université Denis Diderot, Sorbonne Paris Cité, 5 place J. Janssen, 92195 Meudon Principal Cedex, France
\end{abstract} \\ e-mail: frederic.merlin@obspm.fr
}

Received 11 June 2015 / Accepted 31 July 2015

ABSTRACT

\begin{abstract}
Aims. Spectroscopic investigation of the surface of Pluto allows us to constrain the chemical properties of the volatile species of the solar system reservoir. This permits us to obtain the relative abundances of various molecules, their physical properties, as well as their spatial and temporal variation. This also could tell us about the origin of various minor chemical compounds formed during the solar system formation or generated later on by space weathering. This will give us critical information about the evolution processes that may occur in the entire trans-Neptunian objects population, and in particular the biggest objects, which could retain tenuous atmospheres.

Methods. New observations of the surface of Pluto have been carried out along with reanalyses of older observations carried out with the ESO-VLT telescopes and the SINFONI instrument at a mean spectral resolution of 1500 . We present three new near-infrared spectra of Pluto observed at different epochs, and covering the $H$ and $K$ spectral bands showing absorption features of methane, nitrogen, and carbon monoxide ices. We ran different spectral models, based on Hapke theory, to constrain the physical and chemical properties of different sides of Pluto.

Results. We have confirmed the spatial and secular variation of the spectral properties of the surface of Pluto. The abundances, sizes, and temperatures of different ices, such as $\mathrm{CH}_{4}, \mathrm{CO}$, and $\mathrm{N}_{2}$ have been constrained for different parts of the surface of Pluto. The results suggest a temperature probably just above the alpha-beta transition phase of $\mathrm{N}_{2}$ (close to $36.5 \mathrm{~K}$ ), and a probable stratification of the dilution state of $\mathrm{CO}$ and $\mathrm{CH}_{4}$. The presence of minor chemical compounds, such as $\mathrm{C}_{2} \mathrm{H}_{6}$, has been confirmed too, and for data obtained at several sub-Earth east longitudes. Solid $\mathrm{C}_{2} \mathrm{H}_{4}$ is suggested by the spectral modeling with abundance variation following that of solid $\mathrm{C}_{2} \mathrm{H}_{6}$ and solid $\mathrm{CH}_{4}$.
\end{abstract}

Key words. radiative transfer - methods: observational - techniques: spectroscopic - Kuiper belt objects: individual: Pluto

\section{Introduction}

Pluto, a dwarf planet, is known to be the biggest of the transNeptunian objects (TNOs). The TNOs are icy relics orbiting the sun beyond Neptune left over from the planetary accretion disk. These bodies act as unique tracers of the chemical, thermal, and dynamical history of our solar system and are different from one another (Barucci et al. 2008). Although TNOs are considered remnant objects in the solar system, their pristine material has been subjected to space weathering (by UV photolysis, solar wind radiation, cosmic rays, etc., see Strazzulla et al. 2003). Investigating their surface with spectroscopy is a great tool to constrain the chemical properties and evolutionary processes occurring in the solar system, but high signal-to-noise level spectra are required to be able to detect volatile content and constrain their physical properties. Unfortunately, many TNOs are really dark and almost featureless, probably because of the presence of heavily altered materials on their surface. The need is now to focus the investigation on bright objects covered with relatively fresh ices such as Pluto. This object is covered with bright compounds (see, e.g., Owen et al. 1993; Douté et al. 1999) and is a very good candidate, allowing us to retrieve the physical properties of different ices, i.e., their temperature and dilution state. This object also allows us to discover the minor chemical compounds contemporary with the solar system formation and

^ Based on observations made with ESO telescopes at the Paranal Observatory under Program ID. 093.C-0044. to constrain the effects of the space weathering (the formation of organic material via irradiation).

Indeed, previous observations of the brightest TNOs gave us new constraints on the chemical nature and possible evolution of big and cold TNOs. Protopapa et al. (2008), DeMeo et al. (2010), and Merlin et al. (2010) suggest some newly discovered chemical compounds on Pluto, such as ethane and acrylonitrile (both formed from chemical reactions) and confirm the presence of carbon monoxide and nitrogen. Unfortunately, the quality of the spectra were limited and did not enable us to clearly constrain the physical properties and discover other minor species (mainly formed from irradiation of $\mathrm{N}_{2}: \mathrm{CH}_{4}$ : $\mathrm{CO}$ layers); see Moore \& Hudson (2003). Ethane could be formed from irradiation (Hudson et al. 2009) but could be also contemporary with the protosolar nebula, such as $\mathrm{CH}_{4}, \mathrm{~N}_{2}$ and carbon oxides (also found in comets; see, e.g., Bockelée-Morvan et al. 2004; Villanueva et al. 2011). Retrieving the physical properties of ethane ice is essential to be able to constrain the origin of this compound. Indeed, the formation of ethane through natural $\mathrm{UV}$ irradiation is favored in the pure $\mathrm{CH} 4$ matrix rather than in the diluted CH4 in nitrogen (Hudson \& Moore 1997; Brown et al. 2007). The additional search for ethylene, an another natural compound formed by UV irradiation of $\mathrm{CH} 4$ at a similar rate to ethane (Hudson \& Moore 1997; Bennett et al. 2006), may strongly favor the irradiation origin of these hydrocarbons. This is because ethylene is assumed to be marginal compared to ethane and methane in the primordial nebula, because of the rapid transformation of ethylene into ethane via quick ambient 
Table 1. Observational circumstances.

\begin{tabular}{lccccc}
\hline \hline Date & Start time & Exp. time & Long. $\left({ }^{\circ} E\right) /$ Lat. $\left({ }^{\circ} N\right)$ & Airmass $($ analog) & Separation $-\left(\right.$ seeing $\left.^{a}\right)$ \\
\hline 2008.04 .13 & $08: 40$ & $60^{\prime}$ & $5 / 42.5$ & $1.00-1.02(1.02)$ & $\sim 0.65 "\left(\sim 0.35^{\prime \prime}\right)$ \\
2014.07 .17 & $04: 00$ & $55^{\prime}$ & $41 / 50$ & $1.00-1.04(1.04-1.24)$ & $\sim 0.85^{\prime \prime}(\sim 0.5 ")$ \\
2014.07 .22 & $03: 12$ & $55^{\prime}$ & $121 / 49.9$ & $1.00-1.01(1.02-1.15)$ & $\sim 0.85 "\left(\sim 0.55^{\prime \prime}\right)$ \\
2014.08 .17 & $01: 15$ & $55^{\prime}$ & $101 / 49.5$ & $1.00-1.01(1.02-1.16)$ & $\sim 0.90 "\left(\sim 0.55^{\prime \prime}\right)$ \\
\hline
\end{tabular}

Notes. ${ }^{(a)}$ Measured seeing on detector.

hydrogenation, and because it has not been detected in the comas of several comets (Hasegawa et al. 1992; Hiraoka et al. 2000).

Pluto is also surrounded by a thin atmosphere, especially composed of nitrogen with the minor contribution of $\mathrm{CH}_{4}$ and CO (Lellouch et al. 2011). Because of the high eccentricity and inclination of Pluto, the surface temperature and insolated areas change during the whole orbital period of the dwarf planet. Seasonal variation of the atmospheric pressure and the surface ice abundances have been expected and measured by several authors from stellar occultations (Elliot et al. 2003; Sicardy et al. 2003). To constrain the spatial distribution and secular evolution of the main ices on the surface of Pluto, Grundy et al. (2013, 2014) observed this object from 2001 to 2012. They clearly found spatial variation of the $\mathrm{N}_{2}, \mathrm{CH}_{4}$, and $\mathrm{CO}$ physical properties (abundances and/or textures) as well as secular modification. They found that the greatest secular variation occurs on $\mathrm{CH}_{4}$ ice, even if this ice is less volatile than $\mathrm{N}_{2}$ and $\mathrm{CO}$. However, the authors can not firmly state the origin of these secular changes. Spatial variation, change of the viewing geometry, volatile transport phenomenon, or short-term transient changes are possible explanations to interpret their results. They pointed out the need for new observations to give us new insights into this topic. Because of thin spectral features of the main irradiated products in the near-infrared (NIR) range, detection also needs higher signal-to-noise level and good enough spectral resolution. The New Horizons spacecraft (NH), which passed close to Pluto in July 2015, will help us to map and discover several chemical compounds on one side of its surface. The spacecraft's spectral resolution of 300 in the NIR (or 600 in the narrow $2.1-2.25 \mu \mathrm{m}$ range), however, could hamper the detection of the thinnest absorption features, especially those of ethylene or other species provided from the irradiation of $\mathrm{CH}_{4}$ and $\mathrm{N}_{2}$ layers, and give constraints on the temperature and dilution level of $\mathrm{CH}_{4}, \mathrm{CO}$, and $\mathrm{C}_{2} \mathrm{H}_{6}$.

Here, new NIR spectra of Pluto are presented. They have been obtained at a spectral resolution of 1500, which is necessary to better constrain the physical properties of ices known to be present on Pluto's surface $\left(\mathrm{CH}_{4}, \mathrm{C}_{2} \mathrm{H}_{6}, \mathrm{~N}_{2}, \mathrm{CO}\right)$ as well as their distribution, dilution level, and temperature. A portion of the observations have been obtained at almost the same east longitude that was seen by the NH spacecraft, and taken one year prior to the fly-by. The other observations have been carried out at almost the same east longitude as data collected in 2008 with the same instrumental setting. This allows us to check temporal variations and trends found by Grundy et al. (2014) from a survey mainly done during the first decade of the 21th century. Spatial and temporal evolution of the absorption features are presented as well as constraints on the main properties of the ices (abundances, temperatures, and sizes).

\section{Observations and data reduction}

New observations of Pluto have been performed with the Very Large Telescope (VLT) at ESO (Chile). NIR spectroscopy was carried out in July and August 2014 with the Spectrograph for INtegral Field Observations in the NIR (SINFONI ${ }^{1}$ ) installed on the $8.2 \mathrm{~m}$ UT4. SINFONI is an image slicer integral field spectrometer (Eisenhauer et al. 2003; Bonnet et al. 2004) whose field of view is split into 32 image-slitlets, which reflect onto small plane mirrors and is then redirected toward the selected grating. The 32 spectra are then reimaged on a $2048 \times 2048$ pixels Hawaii 2 RG [1-2.5] $\mu \mathrm{m}$ NIR detector. The observations have been performed using the $H+K$ grating (spectral resolution of 1500) covering both $H$ and $K$ bands simultaneously. Table 1 lists the details of the observational circumstances. The chosen plate scale is 100 mas per pixel and observations were carried out in Adaptive Optics (AO) mode, with the AO-loop closed on Pluto itself.

The data reduction was performed with the software GASGANO, version 2.3.3. First the data were corrected for bad lines created from bad pixels located among the four nonilluminated edge pixels at the beginning and end of each row. Master darks, master flats, and bad pixel maps were created within the SINFONI pipeline. We used $\mathrm{Xe}-\mathrm{Ar}-\mathrm{Kr}$ lamps for wavelength calibration, and as part of the SINFONI pipeline, a wavelength map was computed to derive a direct correspondence between pixel position and wavelength. Determining the orientation and position of each spectrum on the detector facilitates the reconstruction of an image cube of the original field of view. Then, the spectra were extracted from the individual data using an IDL script. This script allows us to extract consecutive spectra with several increasing aperture radii (from 1 to 10 pixel-radius, which encompasses the entire FWHM and avoids flux contamination of Charon flux). While the continuum part of the spectrum is obtained from those obtained with the greatest radius, the entire ten consecutive extracted spectra were used to reduce the noise of the individual spectra at each wavelength. A careful inspection guarantees the limitation of the contamination from the flux of Charon using a limiting radius aperture to ten pixels for the new observations obtained in 2014 and those in 2008 (Merlin et al. 2010), which we rereduced for consistency. Contamination of Charon could have strong effects on the shape of the deepest bands of $\mathrm{CH}_{4}$, especially in the $K$ range, where the entire flux of Charon could reach that of Pluto around $2.3 \mu \mathrm{m}$. The flux variation between two consecutive extracted spectra using increasing radius of 1 pixel was checked. The larger aperture was choosen with the constraint that the difference of the entire flux between two consecutive spectra does not reach more than $4 \%$, and that the variation of this ratio does not change by more than $2 \%$, wavelength per wavelength, on the complete [1.45-2.45] $\mu \mathrm{m}$ range. This conservative value guarantees we encompass the maximum flux of Pluto with the lowest contamination of Charon. The Doppler shift was measured and corrected using the JPL Horizons ephemeris server for all observations. The relative speeds between the target and observer have been

1 Information available at http://www.eso.org/instruments/ sinfoni 

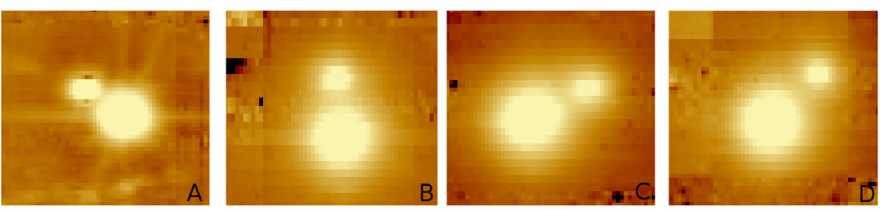

Fig. 1. A) Configuration of Pluto and Charon on April 13th, 2008. B) Configuration on July 17th 2014. C) Configuration on July 22th 2014. D) Configuration on August 17th 2014.

converted in wavelength correction factors for the entire spectra. At the end, and for each night, we computed the final spectrum from the mean value of the entire 11 corrected individual spectra (or 12 for data obtained in 2008). The extraction quality is better for the data obtained in 2008, with a flux difference lower than $2 \%$ between the two consecutive spectra obtained with a 9 and 10 radius aperture. This is thanks to a better seeing, while data obtained near $110^{\circ}$ east longitude in 2014 are more affected by flux contamination. Each configuration is given in Fig. 1.

Two different solar analogs were used to remove the reflected light of the sun and obtain reflectance spectra of Pluto. The two solar analogs are HD 164000 and SA110361, which could be associated with G3V spectral type. Comparison of their color indices in the NIR range reveal that both stars are a bit redder than the sun, the mean spectral slope could reach $+1 \% / 100 \mathrm{~nm}$. In the [1.45-2.45] $\mu \mathrm{m}$ range, a correction using a division of two simple Planck functions (one generated at the temperature of the Sun and one generated at a temperature comprised in the 5200-6400 K range) should efficiently correct for the spectral type and temperature difference of these two stars. The division of the Pluto spectrum by that of HD 164000 or SA110361 give small differences, less than a few percent over the entire wavelength range. There is a major exception in the [1.8-2.0] $\mu \mathrm{m}$ range, mainly due to the airmass difference between both stars and the limited correction of the telluric bands. The fully reduced spectra of Pluto obtained in 2014, converted in reflectance and normalized at $1.5 \mu \mathrm{m}$ are presented in Fig. 2. Spectra obtained at sub-Earth east longitude (SEEL) centered at $101^{\circ}$ and $121^{\circ}$ are quite similar, while those obtained at east longitude centered at $41^{\circ}$ exhibit deeper absorption bands. Then, spectra obtained on July 22th and August 17th have been coadded since they have been taken at the almost same SEEL (around $111^{\circ} \pm 10^{\circ}$ ) and compared to the other spectra obtained in 2008 and 2014 with the same instrumental setting; see Fig. 3. We define geometry on Pluto as in Grundy et al. (2013): "using a right-hand-rule coordinate system in which North is the direction of Pluto's spin vector and East is the direction of sunrise. Zero longitude is defined by the sub-Charon point. Longitudes and latitudes tabulated here were computed by assuming Pluto's spin state is tidally locked to the Buie et al. (2012) orbit of Charon". In this coordinate system, longitude decreases with time.

Figure 2 shows clear identification of the main spectral features of methane ice. The absorption band of pure methane ice is present in all three spectra at $1.69 \mu \mathrm{m}$, as well as the absorption band of solid $\mathrm{N}_{2}$ at $2.15 \mu \mathrm{m}$. The main absorption band of $12 \mathrm{CO}$ appears in the NIR around $2.35 \mu \mathrm{m}$ and the possible one located shortly before $1.58 \mu \mathrm{m}$. The $13 \mathrm{CO}$ band seems to be present at $2.405 \mu \mathrm{m}$. Figure 3 presents slight changes of the spectra obtained at a different east longitude. This mainly affects the depth of the methane ice bands in both $H$ and $K$ ranges. The divisions of the different spectra, to identify the spectral variation dependent on the longitude more clearly, are shown in Fig. 4. Variations of the methane bands depth (located at 1.66,
$1.72,1.79,2.2,2.32,2.375$, and $2.425 \mu \mathrm{m}$ ) are obvious. They are much deeper at smaller SEEL; see Fig. 4 for a comparison with the reflectance spectrum of methane ice. While the spectral behavior of the spectra division is in phase with absorption features of $\mathrm{CH}_{4}$ ice in the $\mathrm{K}$ band, it is not completely the case in the $H$ band, with slight spectral shifts. Dilution of $\mathrm{CH}_{4}$ in nitrogen affects the position and shape of the bands (Quirico \& Schmitt 1997b), so the fact that there is no similar phasage in the $H$ band could be caused by possible variation of the dilution and the physical properties of the methane ice. As weaker bands probe deeper layers, the variation seems to mainly affect the deeper layers. In addition to that, there is evidence of two different spectral slopes between the data obtained near $110^{\circ}$ SEEL and those obtained at $5^{\circ}$ and $41^{\circ}$, with bluer slopes for the last two spectra in the [1.5-1.85] $\mu \mathrm{m}$ and [2.0-2.45] $\mu \mathrm{m}$ ranges. At first order, it seems this slope variation could be mainly due to the broad absorption features of methane ice, but a residual contamination of Charon is not ruled out. Indeed, Charon is covered by huge amount of water ice and the NIR spectrum of $\mathrm{H}_{2} \mathrm{O}$ ice displays deep and broad absorption bands that can affect the whole spectral behavior and slope of Pluto spectra (see the synthetic spectrum of water ice on Fig. 4). For example, the behavior of the spectral division seems to be inversely proportional to the $\mathrm{H} 2 \mathrm{O}$ ice spectral features between 1.55 and $1.72 \mu \mathrm{m}$ (i.e, local spectral minimums of the spectral division when local spectral maximums of the synthetic spectrum of $\mathrm{H}_{2} \mathrm{O}$ ice, and vice-versa). However, this possible contamination appears to be weaker than the effect associated with the methane ice properties variation.

\section{Spatial variation}

The band peak position and fractional band depth of the different absorption features were measured in the $H+K$ range to analyze the spatial variation of the physical and chemical properties of the surface of Pluto. The band peak position is simply identified by the location of the minimum value of the absorption bands. A Monte Carlo test has been performed to compute the mean value and the error (at one sigma level) over 100 spectra that have been generated from each spectrum presented in Fig. 3 . We synthesized each of the 100 spectra from the initial spectrum adding a random value, wavelength per wavelength, comprised within one standard deviation. We used the same method to compute the fractional band depth and its error, following the instruction provided by Grundy et al. (2013) to choose the anchor points (i.e., continuum and minimum of the bands) for comparison purpose. We changed the wavelength window of the second continuum for all the bands $([1.97-1.99] \mu \mathrm{m}$ instead of $[1.98-2.01] \mu \mathrm{m})$ to avoid error due to the telluric bands that were not completely removed during the spectral extraction step and located shortly after $2 \mu \mathrm{m}$. For the bands not reported by Grundy et al. (2013), the equivalent band depth is computed directly from the depth measured at the band peak location, and not from an average computed from a wavelength interval, as done by the cited authors. Measurements of the band peak position of the absorption features do not reveal major wavelength shifts between the three spectra, within the errors, see Fig. 5. The band peak of methane ice bands are usually and marginally less shifted toward shorter wavelength for the data obtained at SEEL $5^{\circ}$, implying a possible lower dilution level of $\mathrm{CH}_{4}$ ice into $\mathrm{N}_{2}$ in this area. In contrast, the fractional band depth of almost all bands are significantly greater at SEEL of $5^{\circ}$ and weaker around $110^{\circ}$, implying a larger amount of pure and less diluted $\mathrm{CH}_{4}$ on the sub-Charon surface. For the bands of $\mathrm{CO}$ and $\mathrm{N}_{2}$, blended in the shoulders of strong $\mathrm{CH}_{4}$ bands, we computed the 
A\&A 582, A39 (2015)
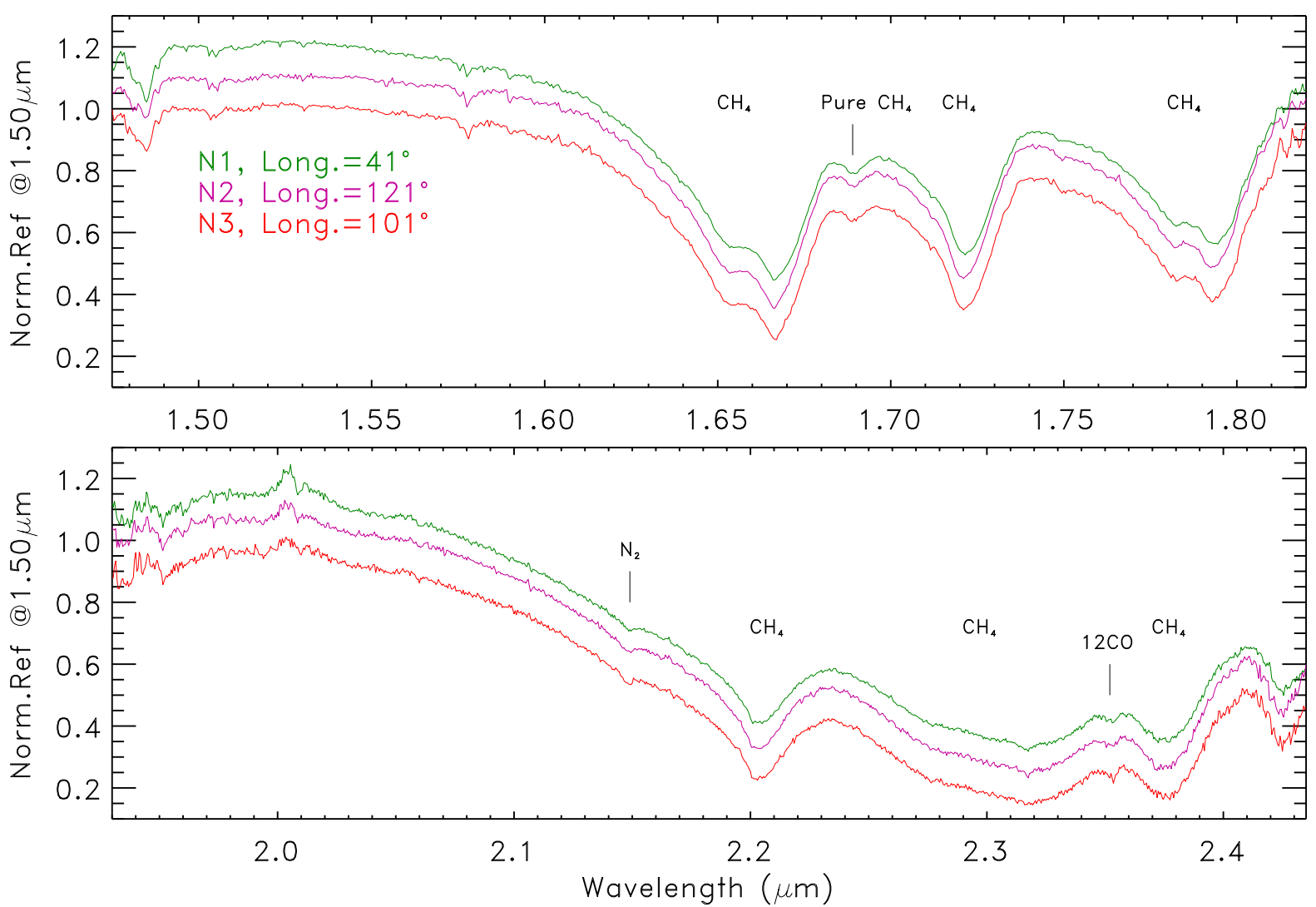

Fig. 2. Spectra of Pluto normalized at $1.5 \mu$ m obtained on July 17th 2014 (in green, shifted by +0.2 in reflectance), July 22 th 2014 (purple, shifted by +0.1 in reflectance), and August 17th 2014 (in red) in the $H+K$ range.
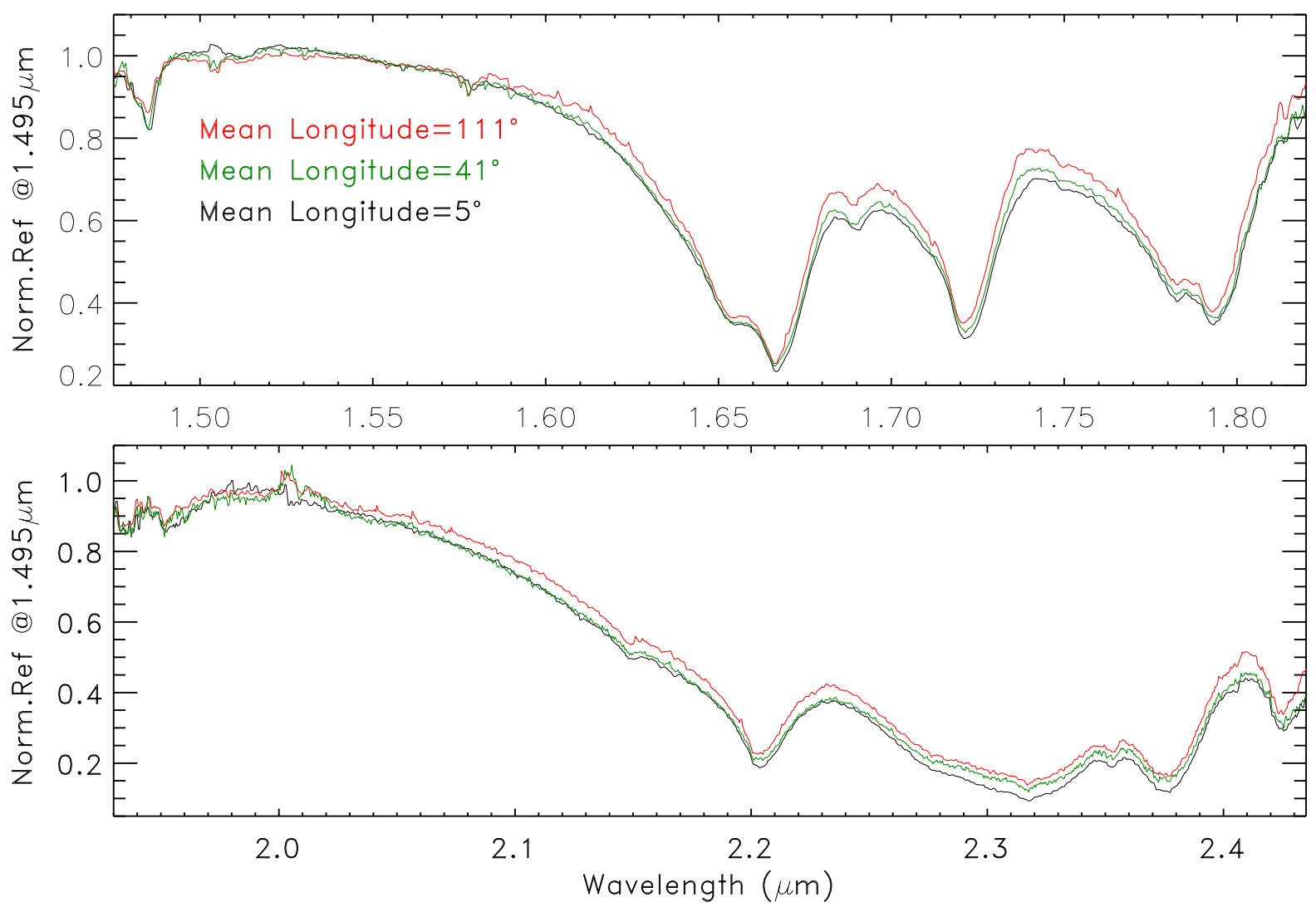

Fig. 3. Reflectance spectra of Pluto normalized near $1.5 \mu \mathrm{m}$, obtained at mean sub-Earth east longitude of $5^{\circ}$ (in black), $41^{\circ}$ (in green), and $111^{\circ}$ (in red). 


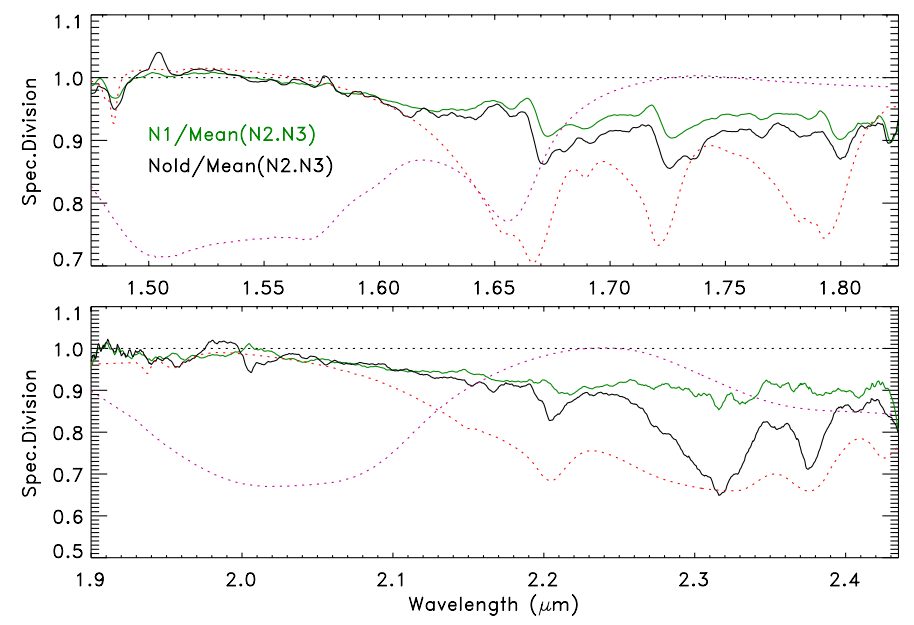

Fig. 4. Division of the normalized spectra presented in Fig. 3. N1, N2, N3, Nold refer to spectra obtained on July 17th $2014\left(\right.$ SEEL $=41^{\circ}$ ), July 22th $2014\left(\right.$ SEEL $\left.=121^{\circ}\right)$, August 17 th $2014\left(\right.$ SEEL $\left.=101^{\circ}\right)$, and April 13th $2008\left(\right.$ SEEL $\left.=5^{\circ}\right)$, respectively. Synthetic spectra of water ice and methane ice have been added (in purple and red dashed lines, respectively).

equivalent band depth in the [2.13-2.165] $\mu \mathrm{m}$ range for $\mathrm{N}_{2}$ and in the [2.3490-2.3560] $\mu \mathrm{m}$ range for $\mathrm{CO}$, with a continuum at the extreme edges of these ranges. This technique should reduce the effect of the $\mathrm{CH}_{4}$ band variation and give an idea of the evolution of the size or texture of these both chemical compounds. Figure 5 presents different trends for these two species, even if the results are not too conclusive for $\mathrm{CO}$, according to the errors. Contrary to the results obtained for $\mathrm{CH}_{4}$, the minimum for $\mathrm{N}_{2}$ appears near SEEL $41^{\circ}$ while results obtained at $5^{\circ}$ and $110^{\circ}$ are quite similar. All these results are compatible with those obtained by Grundy et al. $(2013,2014)$ from observations obtained during the [2000-2012] period.

However, if these trends and conclusions are compatible with those obtained by Grundy et al. (2013), the values of the fractional band depth measured in this work are always greater than those reported by these authors ( 2 to $4 \%$ ). While the increase of the fractional band depth could be possibly explained and validate a secular variation for the data obtained in 2014, this increase is more difficult to explain for the data obtained in 2008, which is covered by the long-term survey. Residual flux contamination of Charon and residual telluric bands could possibly alter measurements of all data. The fact that the wavelength window of the second continuum is not exactly the same could also explain this disagreement to a lesser extent since the continuum level should be almost constant in the whole [1.97-2.01] $\mu \mathrm{m}$ range.

The band peak position of the $\mathrm{CH}_{4}$ bands (see Fig. 5) and the presence of the band located at $1.69 \mu \mathrm{m}$, both indicate that methane cohabits in pure and diluted states on the surface of Pluto. The band peak position of this band (measured at $1.6897 \pm 0.0006 \mu \mathrm{m}$ ) and its shape (well defined) are in agreement with a rather cold methane ice when comparing the results obtained by Grundy et al. (2002). From their laboratory work, they found a band peak position at $1.6895 \pm 0.0003 \mu \mathrm{m}$ at $30 \mathrm{~K}$. This band is slightly blueshifted at $60 \mathrm{~K}$ and almost disappears at $90 \mathrm{~K}$. The profile of the $\mathrm{N}_{2}$ band suggests that this ice is in its Beta phase (see Quirico \& Schmitt 1997b) with a surface temperature greater than $36.5 \mathrm{~K}$. The reported profile and position of the $\mathrm{CO}$ band near $2.3525 \mu \mathrm{m}$ also suggests that $\mathrm{CO}$ is mainly isolated in a solid $\beta-\mathrm{N}_{2}$ matrix. The full width at half maximum
(FWHM) of the band is $4.5 \mathrm{~nm} \pm 1 \mathrm{~nm}$, compared to $1.5 \mathrm{~nm}$ for pure $\mathrm{CO}$ and $3.5 \mathrm{~nm}$ for isolated $\mathrm{CO}$ in $\mathrm{N}_{2}$, and the mean band peak position is $2.3527 \pm 0.0006 \mu \mathrm{m}$, compared to $2.3522 \mu \mathrm{m}$ for isolated CO in $\beta-\mathrm{N}_{2}$ and $2.3517 \mu \mathrm{m}$ in $\alpha-\mathrm{N}_{2}$ (see Quirico \& Schmitt 1997a). Even if the accuracy of the data does not permit us to better constrain the temperature of these ices, all these results suggest pretty cold temperatures, which are probably close to and slightly above the temperature of the $\alpha-\beta$ transition phase of $\mathrm{N}_{2}$ (i.e., above $36.5 \mathrm{~K}$ ).

\section{Spectral modeling}

To confirm the previous analyses of different chemical species and to add constraints on their physical properties, we applied a radiative transfer model based on the Hapke theory (Hapke 1981, 1993). We ran this model to determine the reflectance spectra or the albedo of a medium from individual physical properties of the different chemical components. The albedo is approximated using Eq. (44) of Hapke (1981), i.e.,

$A l b=r 0(0.5+r 0 / 6)+.(w / 8).((1+B 0) P(0)-1)$,

where $w$ is the single-scattering albedo, and $r 0$ the bihemispherical reflectance, which is purely single-scattering albedo dependent. We assume

$r 0=(1-\sqrt{1-w}) /(1+\sqrt{1-w})$,

where $w$ depends on the optical constants and the size of the particles and is computed for a multicomponent surface, assumed to have possible intimate, geographic, and intramolecular mixures (see Poulet et al. 2002; Quirico et al. 1999, for the differences and application in a few cases). We assumed a complex structure with two geographic units A and B, following the work performed by Merlin et al. (2010) on the spectral modeling of the spectra of Pluto. Even if the presence of more than two geographic units exist, different units could be roughly decomposed in two types, with brighter and darker areas (as expected by Buie et al. 2010) and having extremely different chemical properties. The two geographic units are themselves composed of intimate mixtures of several ices. Since part of the methane ice and all the carbon monoxide ice are diluted in solid nitrogen, synthetic intramolecular mixtures of these chemical species have been generated to adjust the best $\mathrm{N}_{2}: \mathrm{CO}$ and $\mathrm{N}_{2}: \mathrm{CH}_{4}$ ratios (see Quirico et al. 1999, for instruction on the procedure). $B 0$ is the ratio of the near-surface contribution to the total particle scattering at zero phase angle and $P$ is the phase function. The method follows the instruction delivered in Merlin et al. (2009), which assumes an albedo approximation model with a phase angle equal to 0 , and $B 0$ is assumed to be close to 0.67 for icy objects (Verbiscer $\&$ Helfenstein 1998). The surface roughness and interferences are negligible. The phase function is approximated by a single Henyey-Greenstein function dependent on the asymmetry parameters $\zeta$ defined as

$$
P(0, \zeta)=\left(1-\zeta^{2}\right) /\left(1+2 \zeta+\zeta^{2}\right)^{1.5} .
$$

The free parameters are the particle size and ratio of the different compounds in the mixture, the ratio between the $\mathrm{A}$ and $\mathrm{B}$ geographic units, the molecular weight of $\mathrm{CO}$ and $\mathrm{CH}_{4}$ in the nitrogen matrix, the asymmetry parameter $\zeta$, and, finally, the temperature of the Planck function correction, which is expected to almost completely correct for the solar analog continuum mismatch. The best-fit models are based on the LevenbergMarquardt algorithm that minimizes the reduced $\chi^{2}$. The minimization is applied outside the telluric absorption bands, which 

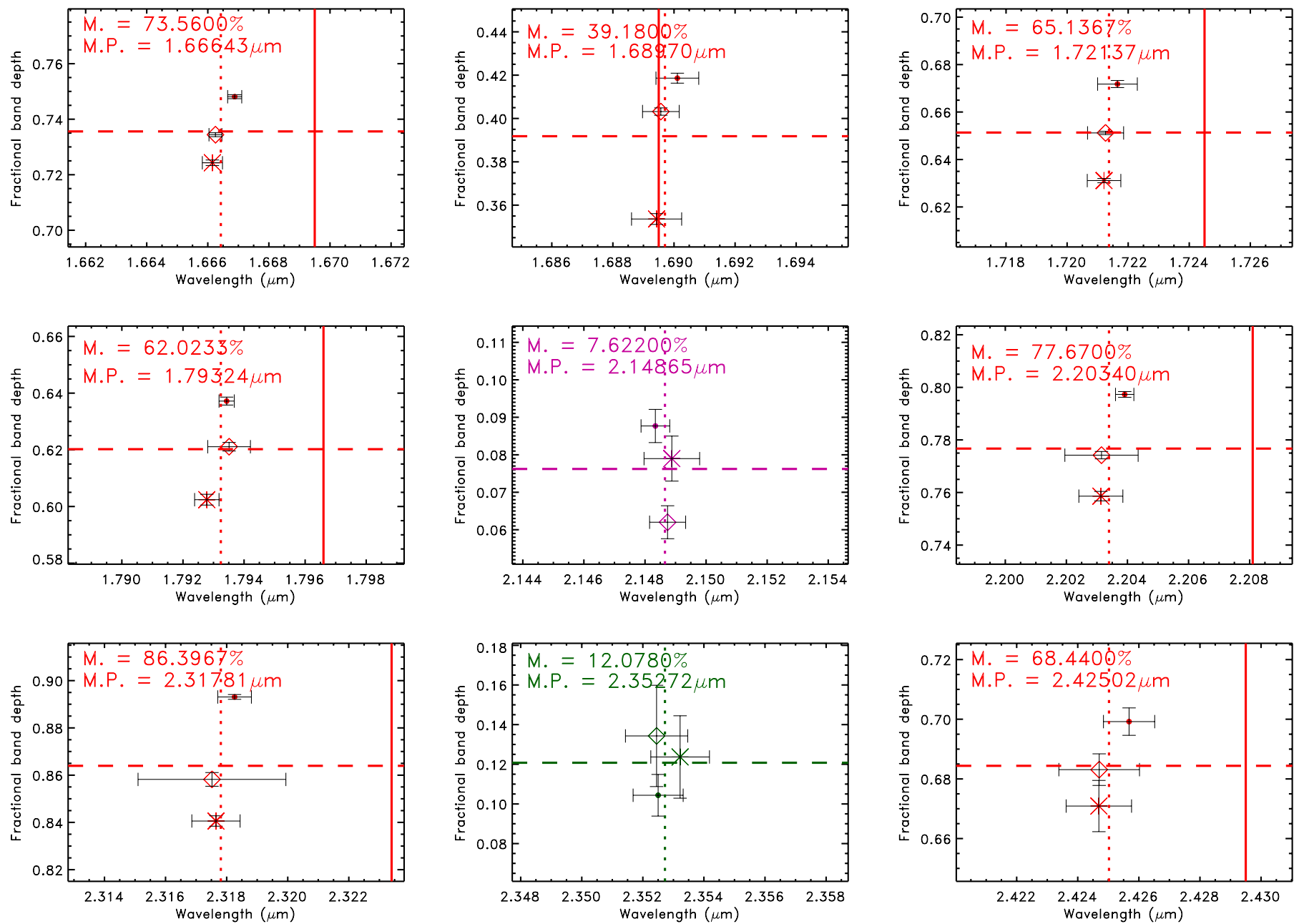

Fig. 5. Band peak location and equivalent band depth of the main absorption features. Band of methane ice is plotted in red, that of $\mathrm{N}_{2}$ and $\mathrm{CO}$, in purple and green, respectively. The results for data at sub-Earth east longitude of $5^{\circ}, 41^{\circ}$, and $111^{\circ}$ are represented with dots, diamonds, and crosses, respectively. Vertical dotted and full lines represent the computed mean band peak position and the position of pure methane ice as reported by Quirico \& Schmitt (1997b) when applicable, respectively. The horizontal dashed line provides the computed mean equivalent absorption band depth. M. and M.P. give the mean fractional band depth and the mean band peak position, respectively.

can affect the results, and the statistical weight of the spectrum is given by computing the standard deviation of the different spectra. The errors made on the synthetic spectra could not be added in the $\chi^{2}$ computation because of the lack of knowledge of the errors of the optical constants. These errors are probably not null but are most likely smaller than those computed from the spectra of Pluto. However, the error on the optical constants are larger in the shallowest bands, which mainly appear in the continuum of the spectra of Pluto exactly where the signal-to-noise level is the greatest. In the strong absorption features, the error on the optical constant should be very small and the limitation on the spectral model itself should prevail at this point. From these considerations, the estimated $\chi^{2}$ is probably a bit greater than those computed, but still accurate enough for comparison purpose. For the models, we adopted the geometric albedo of 0.56 , derived in the $H$ band by Merlin et al. (2010) at SEEL $5^{\circ}$. At larger SEEL, smaller albedo are expected according to the results obtained in the photometric $V$ band by Buie et al. (2010). However, albedo variation in the photometric $V$ and $H$ bands are not necessarily to be the same, and for this reason, the same albedo for all data was adopted. All the optical constants used for modeling are reported in Table 2.
Table 2. Optical constants used in this work.

\begin{tabular}{lcc}
\hline \hline $\begin{array}{l}\text { Chemical } \\
\text { compound }\end{array}$ & Temperature & Reference \\
\hline $\mathrm{N}_{2}$ & 36.5 to 43 K & Quirico \& Schmitt (1997b) \\
${\text { Pure } \mathrm{CH}_{4}}_{\mathrm{N}_{2}: \mathrm{CH}_{4}}^{40 \mathrm{~K}}$ & Quirico \& Schmitt (1997b) \\
$\mathrm{N}_{2}: \mathrm{CO}$ & 36.5 to 43 K & Quirico \& Schmitt (1997b) \\
Titan Tholin & - & Quirico \& Schmitt (1997b) \\
Am. Carbon & - & Khare et al. (1986) \\
$\mathrm{C}_{2} \mathrm{H}_{6}$ & $21 \mathrm{~K}$ & Zubko et al. (1996) \\
$\mathrm{C}_{2} \mathrm{H}_{4}$ & $21 \mathrm{~K}$ & Quirico \& Schmitt (1997b) \\
\hline
\end{tabular}

\section{Results and discussion}

We developed the models, first, using the major chemical compounds as inputs. The main idea is to compare the best-fit models with the previous conclusions and to search for features of anticipated minor compounds that cannot be fitted in these runs. Figure 6 presents the different spectra of Pluto as well as the synthetic spectra generated by the models. Table 3 lists the main 

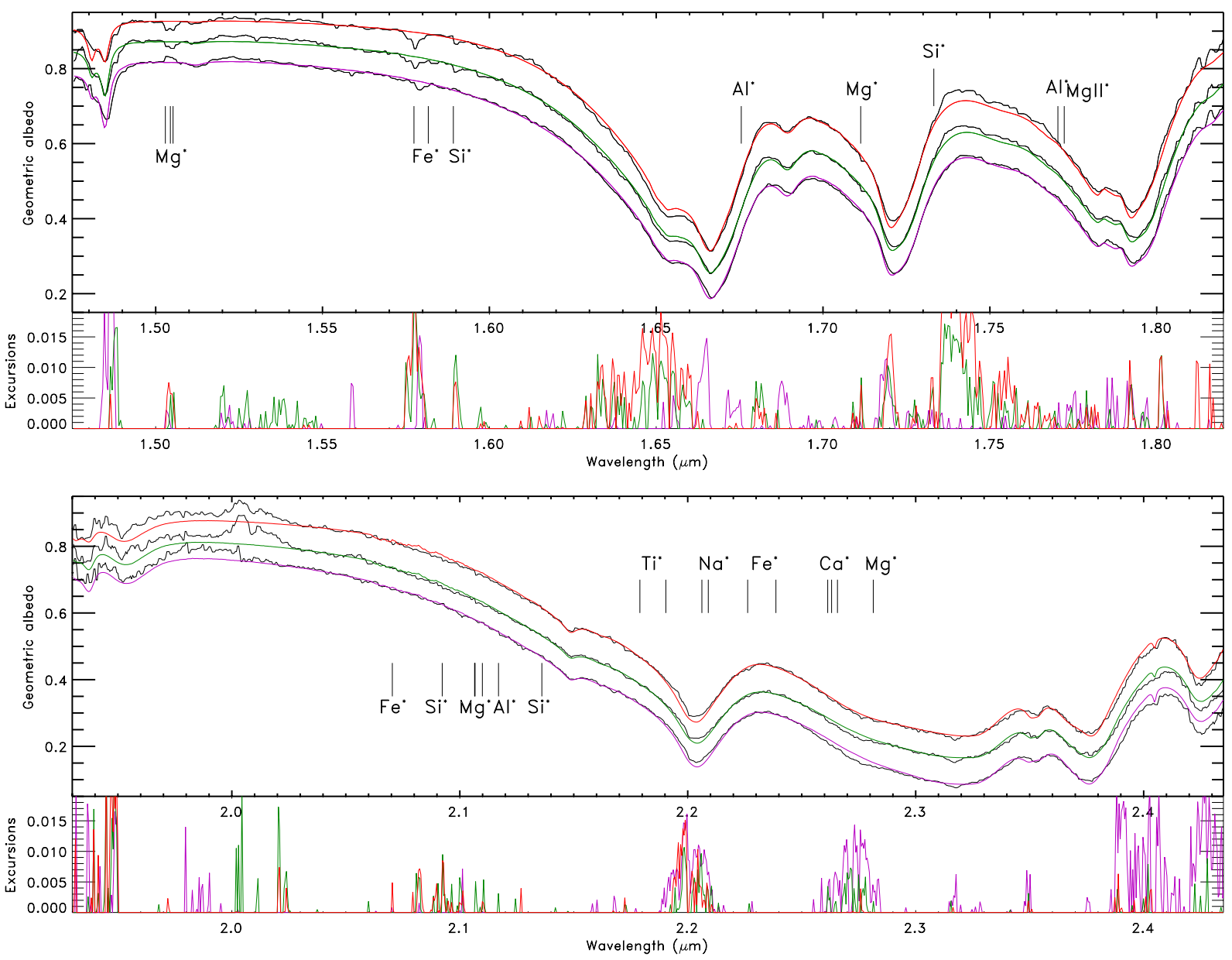

Fig. 6. Results of the spectral modeling. The results are presented for the $H$ and $K$ parts (top and bottom part of the figure, respectively). The top panels show, from the bottom to the top, spectra of Pluto converted in albedo obtained at SEEL of 5,41 , and $111^{\circ}$, respectively. They are associated with their best-fit models, in purple, green, and red (shifted for clarity by $+0,+0.05$ and +0.1 , respectively). The bottom panels present excursions of the models when greater than the observational error defined at $1 \sigma$ level. ${ }^{*}$ Position of metallic lines and their assignment (see Lancon \& Rocca-Volmerange 1992, for more details).

abundances and sizes of the chemical compounds as well as the associated reduced $\chi^{2}$ and the computed asymmetry parameter. Reduced $\chi^{2}$ is rather good as seen from the few and limited excursions of the models outside the $1 \sigma$ error presented in Fig. 6. The abundances and sizes of $\mathrm{N}_{2}, \mathrm{CH}_{4}$, and $\mathrm{CO}$ slightly agree for very constant values at first order. The results agree with a first area composed of a rather thick cm-dm layer of $\mathrm{N}_{2}$ in which $\mathrm{CO}$ and $\mathrm{CH}_{4}$ are diluted, with less than $0.5 \%$ of each constituent embedded in the matrix. As suggested by the models, the second area is mainly composed of pure $\mathrm{CH}_{4}$, close to the mm scale (two different particle sizes are requested here), and an organic compound, such as Titan Tholin. To be specific, the models suggest a greater amount of larger particles of methane near the Charon facing side as well as less abundant diluted $\mathrm{CH}_{4}$ in $\mathrm{N}_{2}$, confirming previous statements. However the trend obtained in nitrogen and carbon monoxide are not confirmed by the models, with the amount and particle size almost constant for $\mathrm{N}_{2}$ and an opposite trend for the abundance of $\mathrm{CO}$. As the result depends on the global $\chi^{2}$, the process favors a better fit of the larger features (i.e., those of methane) rather than the thin features (i.e., such as those of $\mathrm{CO}$ and $\mathrm{N}_{2}$ ).

The excursions of the models outside the $1 \sigma$ error (see Fig. 6) reveal numerous unfitted features as well as a limitation of the fit of a few $\mathrm{CH}_{4}$ bands. These excursions are more numerous in the $H$ band, but usually lower than an albedo difference
Table 3. Main results obtained from spectral modeling, covering the [1.45-2.45] $\mu \mathrm{m}$ range for the three spectra.

\begin{tabular}{lr}
\hline \hline SEEL 5 & \\
red. $\chi^{2}: 1.0068950$ & $\zeta=-0.20742750$ \\
\hline $\mathrm{A}$ area: $25 \%$ & $\mathrm{~B}$ area $: 75 \%$ \\
$\mathrm{~N}_{2}: 98.21 \%(19.2 \mathrm{~cm})$ & $\mathrm{CH}_{4}: 40 \%(3.7 \mathrm{~mm})$ \\
$\mathrm{CO}$ in $\mathrm{N}_{2}: 0.5 \%$ & $\mathrm{CH}_{4}: 38.5 \%(372 \mu \mathrm{m})$ \\
$\mathrm{CH}_{4}$ in $\mathrm{N}_{2}: 0.3 \%$ & $\mathrm{~T}$. Tholin: $21.5 \%(5 \mu \mathrm{m})$ \\
$\mathrm{CH}_{4}: 1 \%(4.4 \mathrm{~mm})$ & \\
\hline $\mathrm{SEEL} 41^{\circ}$ & \\
red. $\chi^{2}: 0.88739985$ & $\zeta=-0.21572792$ \\
$\mathrm{~A}$ area: $24 \%$ & $\mathrm{~B}$ area: $76 \%$ \\
$\mathrm{~N}_{2}: 99.36 \%(19.5 \mathrm{~cm})$ & $\mathrm{CH}_{4}: 38 \%(3.3 \mathrm{~mm})$ \\
$\mathrm{CO}$ in $\mathrm{N}_{2}: 0.3 \%$ & $\mathrm{CH}_{4}: 30 \%(365 \mu \mathrm{m})$ \\
$\mathrm{CH} \mathrm{H}_{4}$ in $\mathrm{N}_{2}: 0.34 \%$ & $\mathrm{~T}$. Tholin: $32 \%(5 \mu \mathrm{m})$ \\
\hline $\mathrm{SEEL} 110^{\circ}$ & \\
red. $\chi^{2}: 0.92165502$ & $\zeta=-0.21666766$ \\
$\mathrm{~A}$ area: $32 \%$ & $\mathrm{~B}$ area: $68 \%$ \\
$\mathrm{~N}_{2}: 99.36 \%(19.5 \mathrm{~cm})$ & $\mathrm{CH}_{4}: 41 \%(2.3 \mathrm{~mm})$ \\
$\mathrm{CO}$ in $\mathrm{N}_{2}: 0.25 \%$ & $\mathrm{CH}_{4}: 20 \%(200 \mu \mathrm{m})$ \\
$\mathrm{CH}_{4}$ in $\mathrm{N}_{2}: 0.39 \%$ & $\mathrm{~T}$. Tholin: $39 \%(5 \mu \mathrm{m})$ \\
\hline
\end{tabular}

of 0.02. In this spectral part, they occur more particularly in the shoulders of the methane bands. The difficulty of fitting these 
bands could be due to the fact that light probes different layers of methane in this wavelength range. As the models do not take stratification or a more complex surface into account, this does not compensate for any dilution or pure variation properties along the light path that affect the band position and shape. The misfit could also be because the dilution level or temperature of methane ice, as obtained in the laboratory, is not compliant with that existing on Pluto. We use a combination of pure and fully diluted methane ice to fit the spectra of Pluto. Protopapa et al. (2015) show that it is possible to generate any dilution level of $\mathrm{CH}_{4}$ in $\mathrm{N}_{2}$ at a given temperature. Unfotunately, their optical constants do not cover the entire wavelength range of the spectra. In addition, this method does not prevent against any potential stratification and/or geographic variation issues, as discussed previously. Analyses performed by Protopapa et al. (2015) provide some information to extract abundance of $\mathrm{CH}_{4}$ in $\mathrm{N}_{2}$ from the blueshift of several bands. From the data collected, it is possible to compare the position of the $v_{2}+v_{3}, v_{2}+v_{3}+v_{4}$ and $2 v_{3}$ bands. The shifts are $+9.2 \pm 0.6 \mathrm{~cm}^{-1},+10.2 \pm 0.7 \mathrm{~cm}^{-1}$, and $+11.2 \pm 1.1 \mathrm{~cm}^{-1}$ for the three bands, respectively, compared to the band position of pure $\mathrm{CH}_{4}$ ice. These blueshifts could be consistent with $15 \pm 6 \%$ of $\mathrm{CH}_{4}$ in $\mathrm{N}_{2}$, assuming no stratification and no geographical variation. Outside the methane bands, there are excursions visible near, for instance, $1.505 \mu \mathrm{m}, 1.575-1.58 \mu \mathrm{m}$, $1.59 \mu \mathrm{m}, 1.71 \mu \mathrm{m}$, and around $1.75 \mu \mathrm{m}$ for most of the three models. These excursions could be partly attributed to the effects of differing stellar absorption lines between the used solar analogs and the Sun with different $\mathrm{Mg}, \mathrm{Fe}, \mathrm{Si}$, and $\mathrm{Al}$ content (see Lancon \& Rocca-Volmerange 1992, for assignment of a few lines, represented in Fig. 6). The feature at $1.58 \mu \mathrm{m}$ could also correspond to a minor $\mathrm{CO}$ band, as suggested by Grundy et al. (2014), DeMeo et al. (2010), or Merlin et al. (2010), especially for the spectrum obtained at SEEL $5^{\circ}$, apparently less affected by the differing stellar lines problem. However, this band has a very weak absorption coefficient compared to the major $12 \mathrm{CO}$ band $(2.35 \mu \mathrm{m})$ and the $13 \mathrm{CO}$ band (located at $2.405 \mu \mathrm{m})$, which are both well fitted by the models. This feature could be because, once again, deeper layers of material are probed. Indeed, the band at $1.58 \mu \mathrm{m}$ is located in the continuum and the light is not strongly absorbed as in the [2.3-2.4] $\mu \mathrm{m}$ range. In this case, this suggests that deeper layers are $\mathrm{CO}$ enriched.

We performed new spectral models in the restricted [2.24-2.42] $\mu \mathrm{m}$ range to follow previous works on the investigation of minor species, where ethane and ethylene both exhibit their strongest absorption features in the wavelength range we covered. The results of these spectral models are presented from Figs. 7 to 9 for each SEEL. The synthetic spectra obtained with ethane really improve models using only $\mathrm{CH}_{4}, \mathrm{~N}_{2}$, and $\mathrm{CO}$. Indeed, most of the excursions obtained with models without ethane and ethylene are removed, or substantially reduced, with models adding ethane. While the presence of ethane seems evident from the results obtained on the computed reduced $\chi^{2}$ (improved by a factor of 2 , at least) or the better agreement of the models presented from Figs. 7 to 9, the presence of ethylene is less obvious. This statement is particularly true at SEEL = $5^{\circ}$, with an almost similar reduced $\chi^{2}$ than that obtained without $\mathrm{C}_{2} \mathrm{H}_{4}$. This could hamper a clear detection of $\mathrm{C}_{2} \mathrm{H}_{4}$ on the surface of Pluto. All models agree within a few percent of both ethylene and ethane on the surface of Pluto. Usually, agreement is better with $3 \%$ to $5 \%$ of each compound, and the models suggest lower $\mathrm{C}_{2} \mathrm{H}_{6}$ and $\mathrm{C}_{2} \mathrm{H}_{4}$ content at greater SEEL (with $5 \%$, $4 \%$, and $3 \%$ found at SEEL $=5^{\circ}, 41^{\circ}$, and $111^{\circ}$, respectively, for both chemical compounds). This result could suggest that the abundance of ethane, and potentially that of ethylene, share

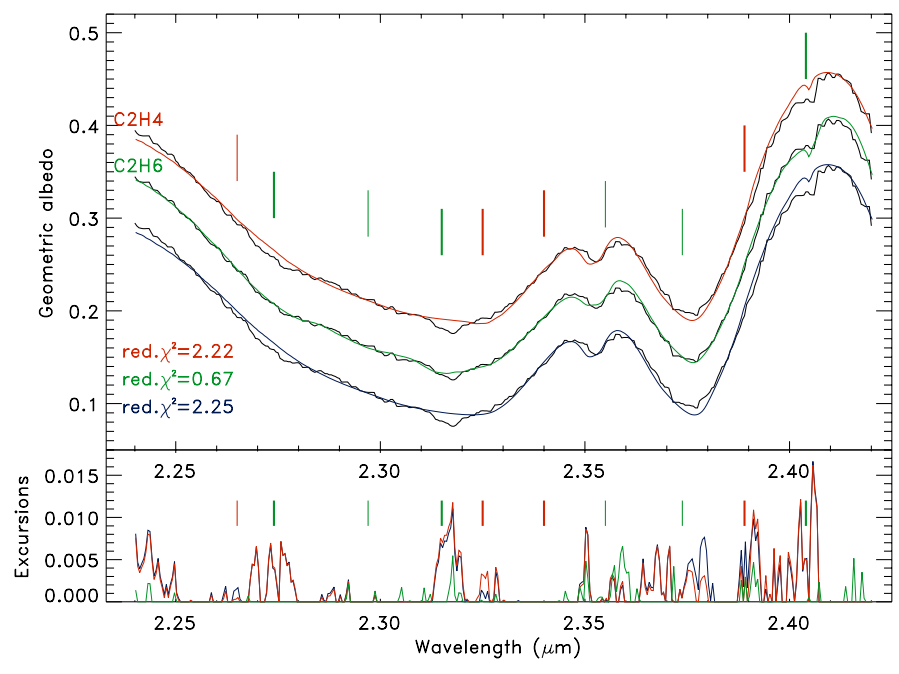

Fig. 7. Results of the spectral modeling in the [2.24-2.42] $\mu \mathrm{m}$ range. In black lines, spectrum of Pluto converted in albedo obtained at SEEL $=$ $5^{\circ}$. From bottom to top, synthetic spectra obtained using the previous model (in blue), using additional ethane (in green), and additional ethylene (in red). These best-fit models have been shifted by $+0,+0.05$ and +0.1 , respectively, for clarity. The bottom panel presents excursions of the models when greater than the observational error defined at $1 \sigma$ level. Vertical segments are located at the band peak position of ethane (in green) and ethylene (in red) features. The thicker segments represent the deepest bands of each chemical compound.

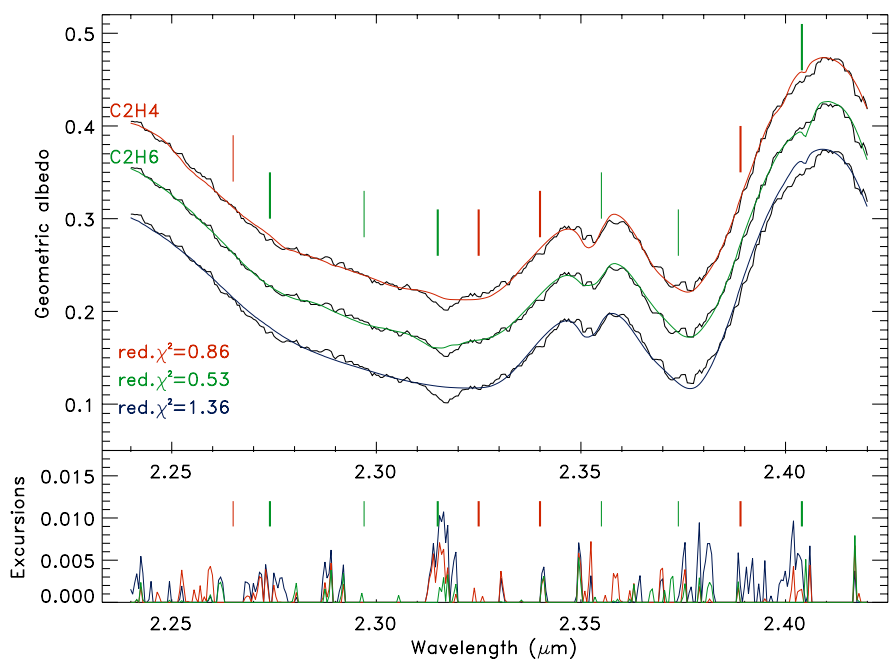

Fig. 8. Same as Fig. 7, except for observations performed at SEEL $=$ $41^{\circ}$.

the same trend as that of $\mathrm{CH}_{4}$. If the fact that both chemical compounds are more abundant in the enriched methane patches is confirmed, this could constrain the origin of these chemical compounds and favors the hypothesis that they are formed from the irradiation of $\mathrm{CH}_{4}$.

From all the models performed, there are a few absorption features that still remain unfitted. The most obvious unfitted bands are located shortly before $2.32 \mu \mathrm{m}$ (close to the deepest absorption band of ethane), near 2.35, 2.375, and $2.40 \mu \mathrm{m}$. The $2.32 \mu \mathrm{m}$ unfitted band could be due to atmospheric methane, with the presence of the deeper $\mathrm{CH}_{4}$ gas absorption feature in this wavelength range centered at $2.317 \mu \mathrm{m}$ (personal communication with E. Lellouch, who detects these features near $1.65 \mu \mathrm{m}$ as well; see Lellouch et al. 2015). A second feature of $\mathrm{CH}_{4}$ gas also exists at $2.371 \mu \mathrm{m}$ and could also partly explain 


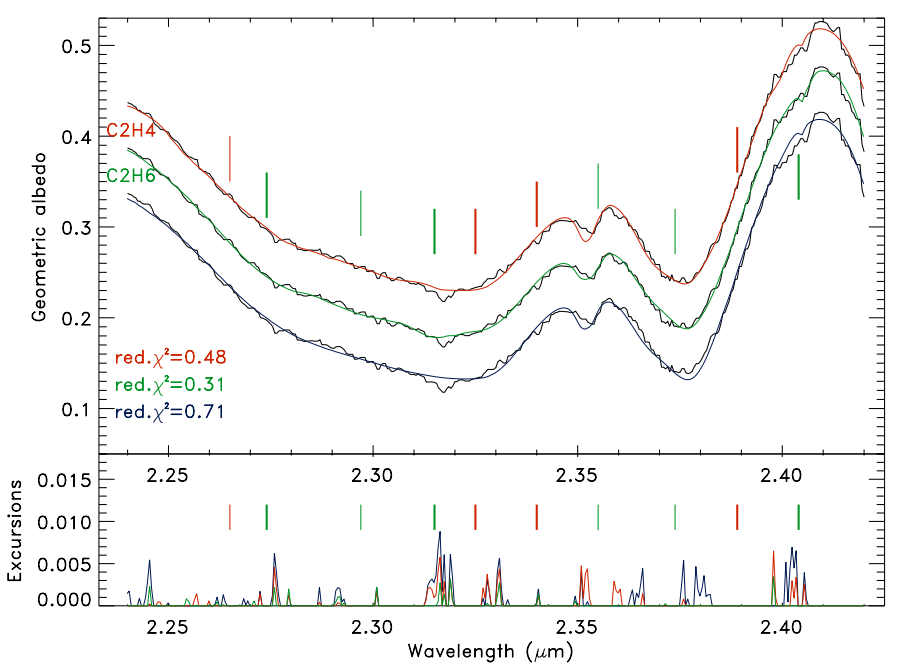

Fig. 9. Same as Fig. 7, except for observations performed at SEEL = $111^{\circ}$.

the mismatch of the model near $2.375 \mu \mathrm{m}$. Higher spectral resolution data are required to state this possibility. At $2.35 \mu \mathrm{m}$ and $2.40 \mu \mathrm{m}$, the blend of $\mathrm{CO}$ and $\mathrm{C}_{2} \mathrm{H}_{6}$ absorption bands are located in the shoulder of strong $\mathrm{CH}_{4}$ bands. This could lead to some diffculty in modeling the first layers of Pluto without consideration of stratification. In addition, the $12 \mathrm{CO}: 13 \mathrm{CO}$ ratio determined from laboratory experiments is not necessarily the same as present on the surface of Pluto. This discrepancy could also limit the capabilities of the models to match the band strength of both $12 \mathrm{CO}$ and $13 \mathrm{CO}$ absorption features at the same time.

\section{Conclusion}

Observations made at different SEEL during 2008 and 2014 give new constraints on the physical state of different ices on the surface of Pluto. A greater amount of larger particles of methane have been suggested near the Charon facing side, as well as less abundant diluted $\mathrm{CH}_{4}$ in $\mathrm{N}_{2}$. This confirms the spatial and secular variation of the spectral properties of the surface of Pluto obtained during the first decade of the 21th century. The temperature of different ices, such as $\mathrm{CH}_{4}, \mathrm{CO}$, and $\mathrm{N}_{2}$ have been constrained for different parts of the surface of Pluto. The results suggest a temperature probably just above the alpha-beta transition phase of $\mathrm{N}_{2}$ (close to $36.5 \mathrm{~K}$ ), and a probable stratification of the dilution state of $\mathrm{CO}$ and $\mathrm{CH}_{4}$, with a higher amount of $\mathrm{CO}$ in deeper layers. The presence of minor chemical compounds, such as $\mathrm{C}_{2} \mathrm{H}_{6}$, seems to be confirmed for several SEEL. Results suggest variation of the abundance or size of this chemical compound that mainly follows the trend observed for $\mathrm{CH}_{4}$ with possible greater amounts of this material near the Charon facing side. Models agree within a few percent of $\mathrm{C}_{2} \mathrm{H}_{4}$, at different SEEL too, but additional observations at higher spectral resolution and higher signal-to-noise level are highly encouraged to firmly state this purpose.

Acknowledgements. The author thanks the reviewer F. E. DeMeo for her suggestions that helped to improve this paper.

\section{References}

Barucci, M. A., Brown, M. E., Emery, J. P., \& Merlin, F. 2008, in Composition and Surface Properties of Transneptunian Objects and Centaurs (The University of Arizona Press), 143

Bennett, C. J., Jamieson, C. S., Osamura, Y., \& Kaiser, R. I. 2006, ApJ, 653, 792

Bockelée-Morvan, D., Biver, N., Colom, P., et al. 2004, Icarus, 167, 113

Bonnet, H., Conzelmann, R., Delabre, B., et al. 2004, in Advancements in Adaptive Optics, eds. D. Bonaccini Calia, B. L. Ellerbroek, \& R. Ragazzoni, SPIE Conf. Ser., 5490, 130

Brown, M. E., Barkume, K. M., Blake, G. A., et al. 2007, AJ, 133, 284

Buie, M. W., Grundy, W. M., Young, E. F., Young, L. A., \& Stern, S. A. 2010, AJ, 139, 1128

Buie, M. W., Tholen, D. J., \& Grundy, W. M. 2012, AJ, 144, 15

DeMeo, F. E., Dumas, C., de Bergh, C., et al. 2010, Icarus, 208, 412

Douté, S., Schmitt, B., Quirico, E., et al. 1999, Icarus, 142, 421

Eisenhauer, F., Abuter, R., Bickert, K., et al. 2003, in Instrument Design and Performance for Optical/Infrared Ground-based Telescopes, eds. M. Iye, \& A. F. M. Moorwood, SPIE Conf. Ser., 4841, 1548

Elliot, J. L., Ates, A., Babcock, B. A., et al. 2003, Nature, 424, 165

Grundy, W. M., Schmitt, B., \& Quirico, E. 2002, Icarus, 155, 486

Grundy, W. M., Olkin, C. B., Young, L. A., Buie, M. W., \& Young, E. F. 2013 , Icarus, 223, 710

Grundy, W. M., Olkin, C. B., Young, L. A., \& Holler, B. J. 2014, Icarus, 235, 220

Hapke, B. 1981, J. Geophys. Res., 86, 4571

Hapke, B. 1993, Theory of reflectance and emittance spectroscopy (Cambridge University Press)

Hasegawa, T. I., Herbst, E., \& Leung, C. M. 1992, ApJS, 82, 167

Hiraoka, K., Takayama, T., Euchi, A., Handa, H., \& Sato, T. 2000, ApJ, 532, 1029

Hudson, R. L., \& Moore, M. H. 1997, Icarus, 126, 233

Hudson, R. L., Moore, M. H., \& Raines, L. L. 2009, Icarus, 203, 677

Khare, B. N., Sagan, C., Ogino, H., et al. 1986, Icarus, 68, 176

Lancon, A., \& Rocca-Volmerange, B. 1992, A\&AS, 96, 593

Lellouch, E., de Bergh, C., Sicardy, B., Käufl, H.-U., \& Smette, A. 2011, The Messenger, 145, 20

Lellouch, E., de Bergh, C., Sicardy, B., et al. 2015, Icarus, 246, 268

Merlin, F., Alvarez-Candal, A., Delsanti, A., et al. 2009, AJ, 137, 315

Merlin, F., Barucci, M. A., de Bergh, C., et al. 2010, Icarus, 210, 930

Moore, M. H., \& Hudson, R. L. 2003, Icarus, 161, 486

Owen, T. C., Roush, T. L., Cruikshank, D. P., et al. 1993, Science, 261, 745

Poulet, F., Cuzzi, J. N., Cruikshank, D. P., Roush, T., \& Dalle Ore, C. M. 2002, Icarus, 160, 313

Protopapa, S., Boehnhardt, H., Herbst, T. M., et al. 2008, A\&A, 490, 365

Protopapa, S., Grundy, W. M., Tegler, S. C., \& Bergonio, J. M. 2015, Icarus, 253, 179

Quirico, E., \& Schmitt, B. 1997a, Icarus, 128, 181

Quirico, E., \& Schmitt, B. 1997b, Icarus, 127, 354

Quirico, E., Douté, S., Schmitt, B., et al. 1999, Icarus, 139, 159

Sicardy, B., Widemann, T., Lellouch, E., et al. 2003, Nature, 424, 168

Strazzulla, G., Leto, G., Gomis, O., \& Satorre, M. A. 2003, Icarus, 164, 163

Verbiscer, A., \& Helfenstein, P. 1998, in Solar System Ices, eds. B. Schmitt, C. de Bergh, \& M. Festou, Astrophys. Space Sci. Lib., 227, 157

Villanueva, G. L., Mumma, M. J., DiSanti, M. A., et al. 2011, Icarus, 216, 227

Zubko, V. G., Mennella, V., Colangeli, L., \& Bussoletti, E. 1996, MNRAS, 282, 1321 